\title{
Dermatology
}

\section{Are There Indications for Isotretinoin Treatment of Hidradenitis Suppurativa?}

\author{
Jurr Boer \\ Department of Dermatology, Deventer Hospital, Deventer, The Netherlands
}

\section{Keywords}

Hidradenitis suppurativa · Isotretinoin · Frictional

furunculoid subtype

I have read with interest the article by Huang and Kirchhof [1], describing an improvement of hidradenitis suppurativa (HS) with the use of isotretinoin, especially in acne-prone younger females with mild disease. This study again raises the question of whether there are indications to treat HS with isotretinoin, and if so, what subgroups of HS are eligible for this treatment. Isotretinoin is well recognized as the gold standard for the treatment of acne, with good long-term results. It is traditionally thought that HS and acne are closely related and because of this isotretinoin has also been tested in HS, as have the related compounds etretinate and acitretin. The durable response rate of HS to isotretinoin reported in one case series of 68 patients and in another study of 358 patients was $16 \%[2,3]$. Because of these case series, the convergent literature data, and the experience of clinicians that its application in HS is often disappointing, the use of isotretinoin has not been recommended in the European Treatment Guidelines for HS [4]. In spite of all these findings, it may be that isotretinoin is still prescribed frequently. This may also be encouraged by the term "acne inversa", as called in the European Guidelines and especially used in the German literature. On the other hand, the HS response rate to another retinoid, acitretin, re-

\section{KARGER}

๑๑ 2017 S. Karger AG, Basel ported in a total of 7 studies of 32 patients, was high (65.6\%) [4]. This fits the concept that there are numerous differences between HS and acne, at least in therapy [5, $6]$, and in addition, that the term acne inversa (as well as HS) appears to be also a misnomer [6]. A possible explanation for the limited therapeutic response of isotretinoin may be the absence of any significant sebaceous gland involvement of HS $[4,5]$.

However, there are patients who respond well to isotretinoin [1-4]. What specific group could this be? It is suggested that the acne-prone HS patients should try isotretinoin [1]. It is well known that acne and HS can occur in the same person. This clinical overlap has led to the inclusion of inhomogeneous patients in the treatment groups. It concerns 2 possible different types of HS: firstly, the classical HS patients (typical lesions on typical locations, i.e., axillary and inguinal involvement) and secondly, the classical HS type with acne affecting the face and the back. The latter disease has also been called acne ectopica or acne tetrad. It has been suggested that patients of these 2 types would respond to isotretinoin in a different way [7-9]. The HS response rates to isotretinoin in a total of 7 studies of at least 11 patients (the number of patients in 2 studies was unspecified) with acne and coexisting HS were at best equivocal compared to the excellent results in acne treatment $[8,9]$. In the same patients the acne seemed to clear completely or was much improved, while in most case reports the HS lesions remained and showed only limited response. A follow-up period was never mentioned [9]. In addition, in the retrospective 
French study of 358 patients with HS, it was found that the presence of acne or a history of previous acne had no influence on the (poor) outcome. Furthermore, isotretinoin has a very poor effect on sinus tracts or tunnels [10], whether located in the convex area of acne or in the inverse area of HS [7-9]. Patients with persistent lesions need surgery deroofing $[7,11]$. So, taking into account the literature as well as the results of the present study [1], I believe that isotretinoin has to be the first step in the treatment of the patient group with both acne and HS with the goal of treating the acne component. The second step will be the treatment of the remaining HS lesions with medications and surgery/deroofing according to a specific HS treatment algorithm, such as the refined Hurley classification [12]. Prior to the treatment with isotretinoin, the patients will need to be told that they can normally expect a cure of their acne but not of their HS.

It is also suggested that patients with mild HS could be isotretinoin responders $[1,2]$. In the present study, mild disease is linked to patients with a Hurley stage 1 classification. However, Hurley staging is static and does not take into account the inflammatory component. In addition, the number of involved anatomical areas is not included [12]. Therefore, a refinement of the current Hurley stage has been proposed. In this way more attention is paid to the differences in disease severity within a Hurley stage. Hurley stage $1 \mathrm{C}$ is considered as serious and is characterized by the presence of migrating lesions; it corresponds with the scarring folliculitis/frictional furuncle phenotype [13]. Recently, it has been suggested to replace this last term with the frictional furunculoid subtype [14].
In this subgroup, the differentiation between fixed and migratory lesions is of great importance, i.e., lesions at fixed locations are suitable for deroofing, while inflammatory lesions at various or staggering locations require anti-inflammatory treatment $[11,12]$. A combination of fixed and migrating lesions is also possible. Patients with inflammatory, migratory lesions are obese and difficult to treat [12-15]. As early as the 1980s, it has been reported that isotretinoin could be useful as an adjunctive therapy in early cases with inflammatory, furunculoid lesions, without tunnels [7]. In addition, in another case series, some indications were found that the response of HS to isotretinoin is more successful in patients with "milder" (furunculoid and papulopustular) disease [2, 9]. Also, in the present study, isotretinoin may be beneficial in patients with this type of inflammatory lesion [1]. Although there seem to be no clear indications for the treatment of HS with isotretinoin, it would be interesting to clarify the true effect of isotretinoin treatment in a specific patient group with only migratory furunculoid lesions.

\section{Key Message}

Patients with both acne and HS being treated with isotretinoin can normally expect a cure of their acne but not of their HS.

\section{Disclosure Statement}

The author declares no conflict of interest. No financial interest exists.

\section{References}

1 Huang CM, Kirchhof MG: A new perspective on isotretinoin treatment of hidradenitis suppurativa: a retrospective chart review of patient outcomes. Dermatology 2017;233:120125.

2 Boer J, van Gemert MJ: Long-term results of isotretinoin in the treatment of 68 patients with hidradenitis suppurativa. J Am Acad Dermatol 1999;40:73-76.

3 Soria A, Canoui-Poitrine F, Wolkenstein P, et al: Absence of efficacy of oral isotretinoin in hidradenitis suppurativa: a retrospective study based on patients' outcome assessment. Dermatology 2009;218:134-135.

4 Zouboulis CC, Desai N, Emtestam L, et al: European $\mathrm{S} 1$ guideline for the treatment of hidradenitis suppurativa/acne inversa. J Eur Acad Dermatol Venereol 2015;29:619-644.

5 Jemec GBE: Long-term results of isotretinoin in the treatment of 68 patients with hidradenitis suppurativa. J Am Acad Dermatol 1999;41:658.
6 Boer J, Nazary M: Long-term results of acitretin therapy for hidradenitis suppurativa. Is acne inversa also a misnomer? Br J Dermatol 2011;164:170-175.

7 Shalita AR, Cunningham WJ, Leyden JJ, et al: Isotretinoin treatment of acne and related disorders: an update. J Am Acad Dermatol 1983; 9:629-638.

8 Jemec GBE: Medical treatment of hidradenitis suppurativa. Expert Opin Pharmacother 2004;5:1767-1770.

9 Boer J: Oral retinoids for hidradenitis suppurativa; in Jemec GBE, Revuz J, Leyden J (eds): Hidradenitis Suppurativa. Heidelberg, Springer, 2006, pp 128-134.

10 Lipsker D, Severac F, Freysz M, et al: The ABC of hidradenitis suppurativa: a validated glossary on how to name lesions. Dermatology 2016;232:137-142.
11 Van der Zee HH, Prens EP, Boer J: A tissuesaving surgical technique for the treatment of mild to moderate hidradenitis suppurativa lesions. J Am Acad Dermatol 2010;63:475-480.

12 Horváth B, Janse IC, Blok JL, et al: Hurley staging refined: a proposal by the Dutch Hidradenitis Suppurativa Expert Group. Acta Derm Venereol 2017;97:412-413.

13 Van der Zee HH, Jemec GBE: New insights into the diagnosis of hidradenitis suppurativa: clinical presentation and phenotypes. J Am Acad Dermatol 2015;73:S23-S26.

14 Boer J: Should hidradenitis suppurativa be included in dermatoses showing koebnerization? Is it friction or fiction? Dermatology 2017, Epub ahead of print.

15 Hampton P, Meggitt S: Better definition of hidradenitis suppurativa subtypes is needed to progress disease-specific treatment outcomes. Br J Dermatol 2017;176:847-865. 